\title{
Research on and Application of Digital Design Framework of Full-coverage Crowns and Bridges Denture
}

\author{
Guodong Yan ${ }^{1, a}$, Guangyun $\mathrm{Ke}^{1, \mathrm{~b}}$ \\ ${ }^{1}$ School of Mechanical Engineering, Nanjing Institute of Technology, Nanjing 211167, China \\ aygd0537@126.com, b280490577@qq.com
}

\begin{abstract}
Keywords: Full-coverage crowns and bridges denture, Skin subdivision, Bidirectional sewing Abstract. Digital restoration of the full-coverage crowns and bridges mainly consists of the connector generating and model fusion. Through defining the section template, the default connector can be generated under the guide of the idea of skin, whose curve can be edited by the control points. If the connector meets the requirements, the connector's boundary should be projected onto the adjacent full-coverage crowns or bridges. The inner regions are tailored by the projection curve, and finally the full-coverage crown, bridge and connector are fused by the mean of bidirectional sew algorithm. And the restoration of the full-coverage crowns and bridges denture can be obtained by smoothing the transition area. It's good quality, high efficiency and can meet the clinical demand.
\end{abstract}

\section{Introduction}

With the development of the modern dental and CAD/CAM technology, digital design has been widely used in oral cavity repair, which has the advantages of convenience and high efficiency, short cycle and high precision ${ }^{[1]}$. In oral clinical rehabilitation, if one or more adjacent tooth missing, digital restoration of full-coverage crowns and bridges, mainly including connector generating and the models fusion, is commonly used. Digital restoration of full-coverage crowns and bridges is universally used in foreign commercial oral software, while there is little domestic research in China. Yuchun Sun et al use Surface 10.5, a reverse engineering software, to design the framework of PFM fixed partial denture of back teeth ${ }^{[2]}$. Yali Song ${ }^{[3]}$ tries to restore the morphology of the full-coverage crowns by Nurbs surface, they expand the surface along the medial and distal direction to intersect with the bridge, obtaining full-coverage crown bridge model by Boolean operation. The above design is aid with the general reverse engineering software, and the operation is complicated. In view of this, digital design of the basal crowns is studied by $\mathrm{Tao}^{\mathrm{An}}{ }^{[4]}$, and a special prototype system has been developed. In the system, overall deformation of the connector can be realized by inputting parameters, while local deformation can't, which reduces the editability of the connector. A complete design framework of full-coverage crowns and bridges denture is proposed in this paper, which contains connector generating and model fusion. It meet the demand of the function of the fullcoverage crowns and bridges denture well. What's more, it is efficient and easy in operation.

\section{Generating the Connector Surface}

After the model design of full-coverage crowns and bridges, they are still independent. Only connecting them together to form a unified whole, the numerical controlled manufacturing can be done. Therefore, a design strategy of connector based on skin subdivision is proposed in this paper. First, the section template curve defined is projected to the models of the full-coverage crown and bridge. Second, ridge line is structured between the two curves under the guidance of the idea of skin, and it is divided into triangular mesh ${ }^{[5]}$. This design strategy avoids the processing of the compatibility of section line, and it's efficient.

Define Cross Section Template. Teeth is mainly divided into four categories, that is incisor (also known as the front teeth), fangs, premolar and molar. According to the surface of medial and distal teeth, three section templates of connector are defined, they are round, oval and triangle with transition circular arc, as shown in Fig.1. The section template, connector's body boundary 
constraints, determines the initial shape of the connector ${ }^{[6]}$. In order to improve the editability of the connector, some points from the section template are chosen as control landmark (points on the section template shown in Fig.1). If not satisfied with the default shape of connector, it can be adjusted through the control points.
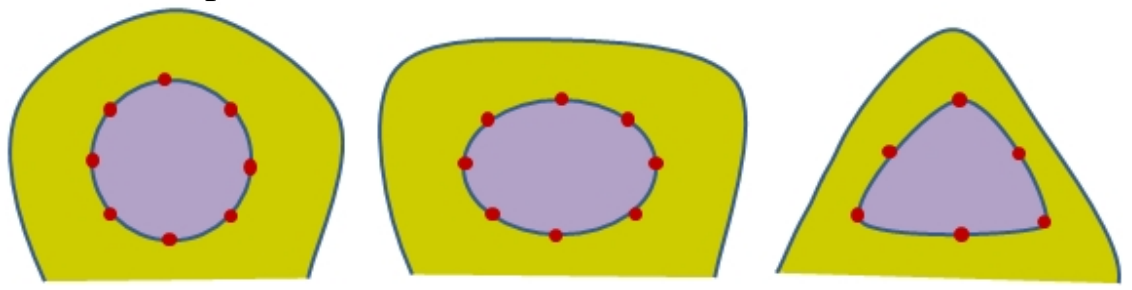

Fig.1 Three section templates of connector

When building the mesh surface of the connector, the appropriate section template should be selected firstly, then the corresponding control landmark will be projected on the full-coverage crown or bridge model to generating B spline curve. Finally, the B spline curve should be projected into the model as the boundary edit curve of the connector. According to the profile form of the denture restoration, the heuristic selective method is chose to call section template. If the tooth is incisor or fang, choose triangular section template. If it's premolar or molar, the bounding box of the model should be calculated. According to the height and width ratio of the surface of the bounding box, the section template is determined.

Skin Subdivision Algorithm. Two boundary curves has been obtained by section template projection algorithm. Then the ridge line can be built between the two boundary curves under the guidance of ideas of skin, through uniform discretization of the ridge line, triangular mesh is obtained by mesh subdivision surface. To build the ridge line, the most important process is determining the endpoint of ridge line, which can be intercepted on the boundary curve by means of sampling. In order to make the surface mesh density of the connector consistent with that of the full-coverage crowns and bridges model, sampling step is half of the sum of the average length of the full-coverage crowns and bridges model mesh.

Suppose the edge set of the full-coverage crown is $E d g e^{c}=\left\{e_{1}^{c}, e_{2}^{c}, \ldots e_{n 1}^{c}\right\}$, the edge set of the bridge is $E d g e^{p}=\left\{e_{1}^{p}, e_{2}^{p}, \ldots e_{n 2}^{p}\right\}$, in these condition, the average mesh length is:

$$
\text { len ave }=\left(\frac{1}{\mathrm{n}_{1}} \sum_{\mathrm{i}=1}^{\mathrm{n}_{1}} \mathrm{e}_{i}+\frac{1}{\mathrm{n}_{2}} \sum_{\mathrm{j}=1}^{\mathrm{n}_{2}} \mathrm{e}_{j}\right) / 2
$$

Uniform sampling is carried out on one of the projection curve in the average mesh length. Take the full-coverage crowns model as an example, and take as the sampling length. Starting from the first point of the boundary curve, sampling sequence will be obtained: $\operatorname{Sec} V t^{c}=\left\{v t_{1}^{c}, v t_{2}^{c}, \ldots v t_{k}^{c}\right\}$.Then, taking $S e c V t^{c}$ as a reference point sequence, a clipping point sequence $\operatorname{Sec} V t^{m}=\left\{v t_{1}^{m}, v t_{2}^{m}, \ldots v t_{k}^{m}\right\}$ will be obtained by intersecting on the boundary curve of the bridge model ${ }^{[7]}$.

If only use two boundary curve of the skin surface subdivision to get connector's mesh, the connector will lack editing flexibility. Thus, a transition curve is added between the two boundaries, and the clipping point sequence can take the halfway point clipping point sequence of the two boundaries:

$$
v t_{i}^{m}=\left(v t_{i}^{c}+v t_{i}^{p}\right) / 2, \quad i=1,2, \ldots k
$$

Thrice B spline curve is chosen as the expression form of ridge line. Points, that the serial number is corresponding to clipping point sequence, is chosen as a set of interpolation points, as shown. As Fig. 2 shown, discrete points are connected between two adjacent ridges, and the triangular mesh subdivision surface is obtained. 


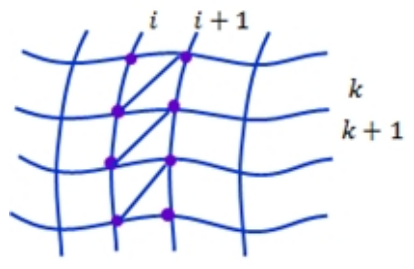

(a) mesh generation

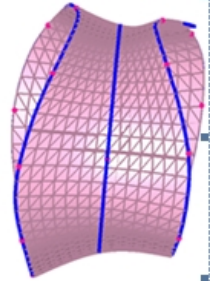

(b) mesh surface of the connector

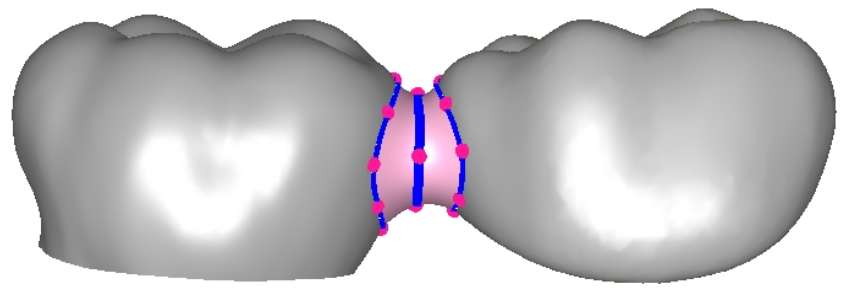

(c) overall effect of the connector

Fig.2 Mesh clipping of the connector.

Editing of the Connector. On the section templates, several control landmark are selected as the editor control points of the curve. A new B-spline curve is generated by moving the control landmark on the surface of the restoration, and projection curve whose shape is changed is obtained by projecting it to the surface of the restoration. Then regenerate the connector's mesh surface according to the skin clipping algorithm, and editing the connector's surface can be realized. Take the connector of the molar's crown and bridge for example, Fig. 3 shows transition effects of the connector's shape, and Fig.4 shows the effects for the connector's shape by the middle transition curve.
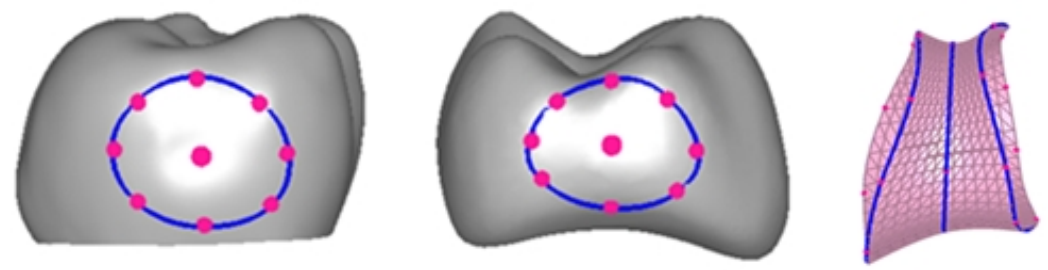

(a) Original connector's surface
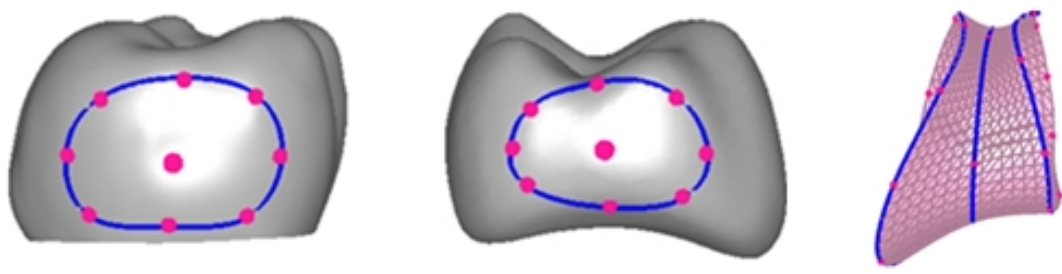

(b)Connector's surface after editing

Fig.3 Mesh surface of the connector.
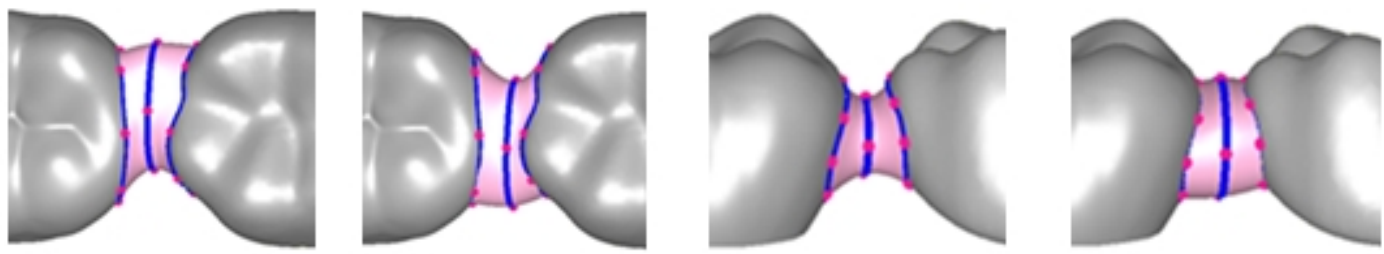

Fig.4 Edit the shape of the connector

\section{Mesh Fusion Algorithm}

Mesh pre-processing Before mesh fusion, because the connector boundary and model of the full-coverage crown and bridge fit sophisticatedly, there is no boundary for mesh sewing. Therefore, 
mesh pre-processing must be carried on to create the conditions of the mesh sewing. Method is that use the boundary curves from the connector generating to cut out a hole on the surface of the model of the full-coverage under the guide of mesh cutting algorithm ${ }^{[8]}$, as shown in Fig.5.

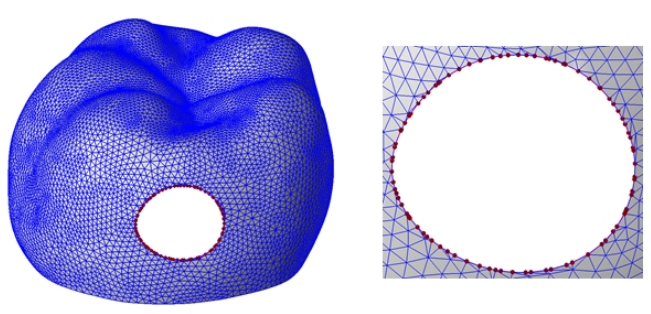

Fig.5 Mesh pre-processing

Mesh sewing .The concept of shape factor is introduced in this paper shape factor, suppose the length of the triangular piece is $e_{1}>e_{2}>e_{3}$, that the shape factor is:

$$
\lambda_{t r i}=\frac{e_{2}+e_{3}-e_{1}}{e_{1}}
$$

Suppose the stitching mesh is $M e s h^{s}$, steps of mesh bidirectional sewing based on shape factor are shown in Fig.6.

(1) Query the boundary vertexes, set them as $B n d P t^{1}=\left\{p t_{1}^{1}, p t_{2}^{1}, \ldots p t_{n}^{1}\right\}$ and $B n d P t^{2}=\left\{p t_{1}^{2}, p t_{2}^{2}, \ldots p t_{m}^{2}\right\}$ respectively.

(2) Take the first vertex $p t_{1}^{1}$ in $B n d P t^{1}$ as the initial point, then calculate nearest vertex with $p t_{1}^{1}$ in $B n d P t^{2}$, and take it as $p t_{j}^{2}$. Query the adjacent vertices with $p t_{1}^{1}$ and $p t_{j}^{2}$, and they can built four candidate triangles shown in Fig (a).

(3) Calculate the shape factor of the four candidate triangles and compare the values, and inserted the candidate triangular with the biggest shape factor into the topology of $\mathrm{Mesh}^{s}$.

(4) Take the outside of the two edges which connect $B n d P t^{1}$ and $B n d P t^{2}$ as reference edge, such as $p t_{1}^{1} p t_{j-1}^{2}$ and $p t_{1}^{1} p t_{j}^{2}$ shown in Fig (b). Extend a boundary point on each side, and construct another four candidate triangles. Calculate the shape factor of the candidate triangles, and insert the candidate triangular with the biggest shape factor in the topology.

(5) Cycle step (4), the triangles will clip on both sides according to the value of the shape factor. When vertexes in $B n d P t^{1}$ and $B n d P t^{2}$ are exhausted, use the last two vertexes in the exhausted side to build a triangular and mesh will be closed.

(6) Update the topology, connect the suture meshs, restoration model and connector together to build a unified mesh model. Transition is smooth in suturing border by Laplace, which eliminates stress concentration $^{[9]}$. Mesh sewing effect is shown in Fig.7.

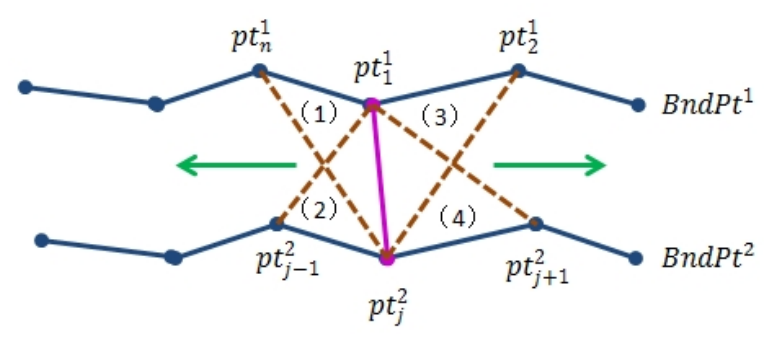

(a) Initial candidate triangulars

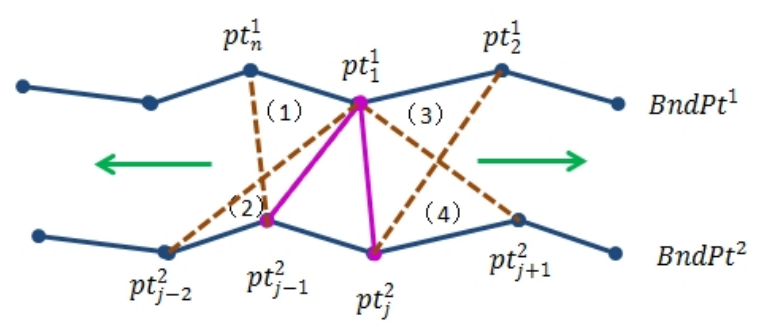

(b)Bidirectional clipping of triangulars

Fig.6 Principle diagram of the Bidirectional clipping. 


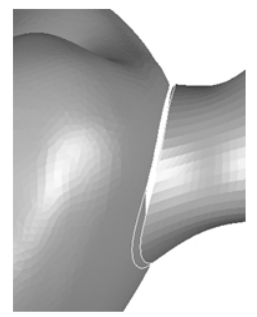

(a) Pretreatment model

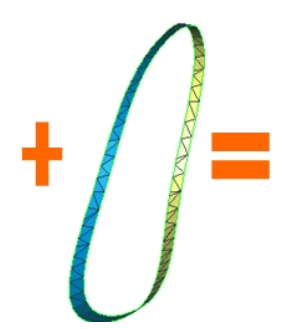

(b) Suture mesh

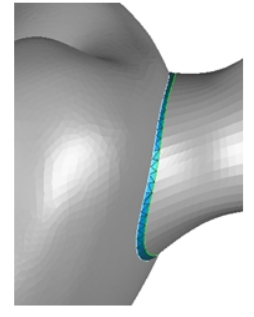

(c) Model combining

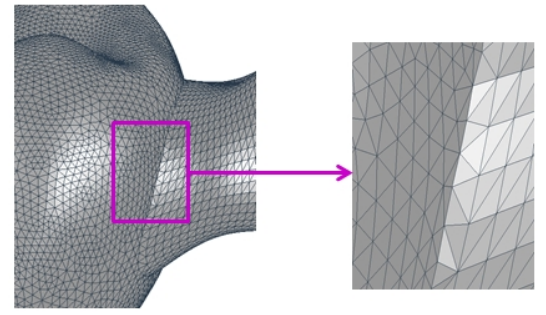

(d) Mesh model after combining

Fig.7 Mesh fusion.

\section{Algorithm Validation and Analysis}

As shown in Fig.8, the full-coverage crown bridge is taken as an example to verify the validity of the algorithm. Date of mandibular molar need for full-coverage crowns and bridges repair is shown in Fig.(a). As shown in Fig.(b), after the design of full-coverage crowns and bridges, the connector is needed to connect them together. Fig.(c) shows the mesh model by calling the section template. Because its overall position lies on the up and cross-sectional area is small, it exists strength deficiencies. Therefore, control points on the boundary curve are adjusted to edit the shape of the connector, until it meets the requirements, as shown in Fig.(d). Then, pretreatment of the full-coverage crowns and bridges is done, and the inner regions of the boundary curve is deleted, which creates boundary conditions for the mesh fusion, as shown in Fig.(e). Finally, three parts of the full-coverage crowns and bridges denture is fused under the guide of the mesh bidirectional sewing algorithm based on shape factor, as shown in Fig.(f). Fig.(g) shows the overall effect of the full-coverage crowns and bridges restoration, the size of each part is coordinated, the form is complete, and the joint is smooth. It already can be used in digital processing and manufacturing of the model.

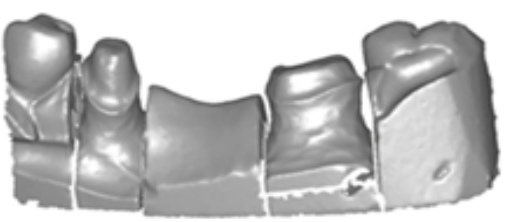

(a) Original model

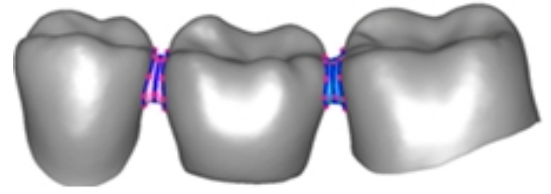

(c) Surface of the original connector

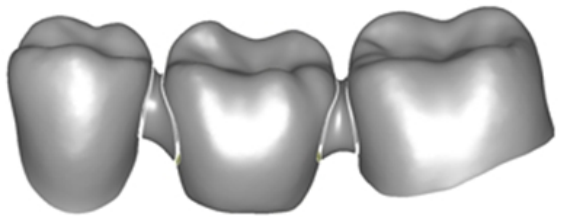

(e) Model pretreatment

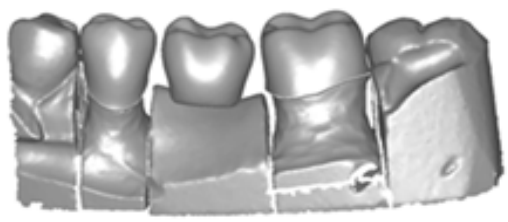

(b) Design of the full-coverage crowns and bridges

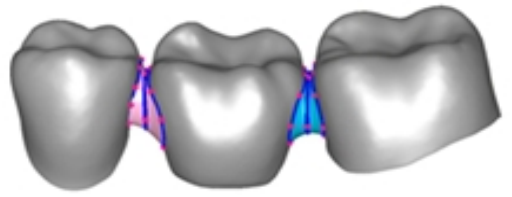

(d) Edit the surface of the model

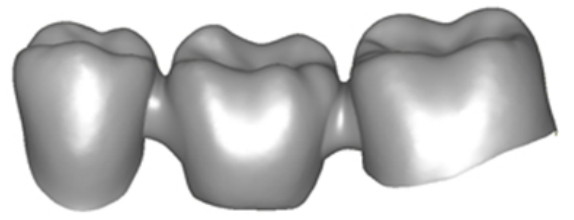

(f) Mesh fusion

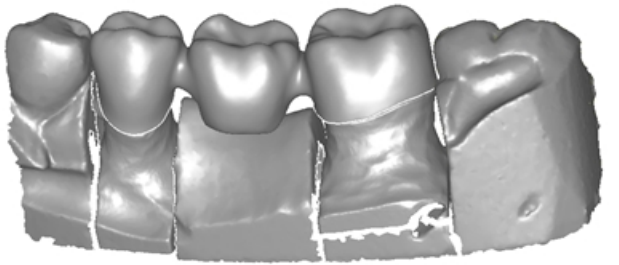

(g) Actual effect of repair

Fig.8 Design process of the full-coverage crowns and bridges. 
Three full-coverage crowns and bridges are designed by the algorithm in this paper, 3 Shape and EXOCad of commercial dental CAD/CAM system, the result is shown in Fig.9. By analysis and comparison, full-coverage crowns and bridges designed by the algorithm in this paper is better. Mesh triangles generated by 3Shape and EXOCad system is long and narrow, while those generated by algorithm in this paper are uniform distribution. Especially in the fusion zone (red box area), triangular mesh on both sides of the model have no obvious difference in shape and size. The connector boundary keep smooth, and the stress concentration phenomenon is eliminated.

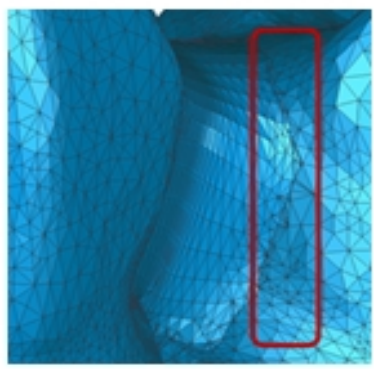

(a) 3Shape

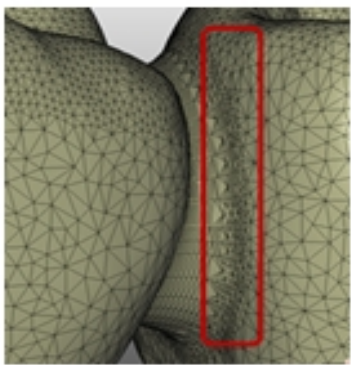

(b) EXOCad

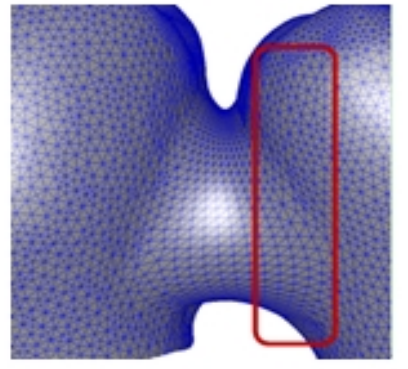

(c)Algorithm in this paper

Fig.9 Full-coverage crowns and bridges designed by different algorithms

\section{Conclusion}

Digital design of the full-coverage crowns and bridges is a systematic project. After the design of the full-coverage crowns and bridges, a connector is needed to fuse them together, forming a complete digital model. In this paper, digital design framework of the full-coverage crowns and bridges is proposed based on the ideas of skin. Compared with the traditional algorithm, this algorithm does not need to do compatibility of the ridge lines, and the efficiency of the algorithm is improved. The full-coverage crown and the bridge is connected under the guide of bidirectional sewing based on shape factor. Restoration designed by digital design framework of full-coverage crowns and bridges denture is high efficiency and good quality. It can satisfy the need of clinical dental restorations.

\section{Acknowledgements}

The work is supported by National Natural Science Foundation of China (NO.51405234), the Natural Science Foundation of Jiangsu Province of China (NO: BK20131341 and BK20150728) and Open Fund of Key Construction Disciplines of School of Mechanical Engineering, Nanjing Institute of Technology (JXKJ201514).

\section{References}

[1] Mingming $\mathrm{Xu}$, Feng Liu. CAD/CAM technology in the application of the oral cavity repair [J]. Chinese Journal of Practical Stomatology, 2013, 6(6):321-326. (In Chinese)

[2] Yuchun Sun, Peijun Lv. Computer aided design for fixed partial denture framework based on reverse engineering technology [J]. Chinese Journal of Stomatology, 2006, 41(3):175-177. (In Chinese)

[3] Yali Song, Jia LI, Ping Gao, et a1. The Feature-based Design of Dental prosthetic Fixed Bridge [J].Modular Machine Tool \& Automatic Manufacturing Technique, 2006, 11:16-23. (In Chinese)

[4] Tao An. Research on and Application of Oral Basal Bridge Digital Design Technology [D]. JiangSu: Nanjing University of Aeronautics and Astronautics, 2008. (In Chinese)

[5] Jingqiao Zhang. Subdivision Surface and Its Application in Surface Modeling Research [D]. Zhejiang: Zhejiang University, 2003. (In Chinese) 
[6] GuoDong Yan, Ning Dai, Wenhe Liao, et a1. The Digital Design of the Fixed Bridge Connector Based on Strength Constraint [J]. Manufacture Information Engineering of China, 2012(12):42-45. (In Chinese)

[7] Gossard S D C. Automatic Triangular Mesh Generation of Trimmed Parametric Surfaces for Finite Element Analysis [J]. Computer Aided Geometric Design, 1998, (3):199-222.

[8] Taskonak B, Mecholsky JJ Jr, Anusavice KJ. Fracture surface analysis of clinically failed fixed partial dentures. J Dent Res. 2006, 85(3):277-281.

[9] Ma Y, Li Z. Consistence segmentation of triangle mesh using Laplace spectral embedding and Mean Shift [J]. Journal of Image \& Graphics, 2012, 17(10):1292-1297. 